\title{
The Emotion of Texture Under Different Senses
}

\author{
Qiao Jie ${ }^{\text {a }}$, Duan Yiting ${ }^{\text {a, }}{ }^{1}$, Zhang Yichi ${ }^{\mathrm{b}}$ \\ ${ }^{a}$ College of Textile Science and Engineering (International Institute of Silk), \\ Zhejiang Sci-Tech University, Hangzhou, CN \\ ${ }^{\mathrm{b}}$ School of Art and Design, Zhejiang Sci-Tech University, Hangzhou, CN
}

\begin{abstract}
In order to explore the effect of different textures on human emotions under different sensory conditions, we collected the effective tendency of textures of six types from 18 to 30 years old subjects in visual, tactile and auditory experiments through psycho-physical experiment. The results show that the subjects have different emotional feelings for textures under different senses. But in all experiments, wood is a relatively safe and reliable textures in subjects' cognition. This essay, through the study of textures, aims to raise design suggestions on the use of large medical equipment.
\end{abstract}

Keywords. Texture, Emotion, Different sensory

\section{Introduction}

A common use of texture was to add interest to a monochrome boring space. Texture describes the material used by an object, which is something that can be touched, felt or processed ${ }^{[1]}$. Akamatsu and the team using a target selection task under different sensory feedback conditions ('normal', auditory, color, tactile, and combined) found that tactile feedback was the quickest ${ }^{[2]}$. Although the tactile system could well recognize familiar objects, Klatzky et al. believed that the tactile perception of forms was not always accurate and effective ${ }^{[3]}$. For the wood texture, visual stimulation depends on the presence of wood nodes, colors, structure, and treatment of indoor wood surfaces; auditory stimulation strongly depends on individual sensitivity; Tactile stimulation depends on the heat flux of the material ${ }^{[4]}$. Compared with artificial materials, Nyrud and Bringslimark pointed out that tactile contact with wood would not cause psychological or physical stress response ${ }^{[5]}$. Similarly, Bhatta et al. 's study showed that natural and smooth wood surfaces were more positively perceived in terms of emotional touch ${ }^{[6]}$.

As there is less research on the impact of texture on human emotion under different senses at this stage, this paper focuses on a few specific psycho-emotional words to explore the specific impact of texture on human emotion. The results could be used as a starting point to improve the appearance of some large medical equipment, by changing the external texture to achieve the purpose of alleviating the negative emotions of the user.

\footnotetext{
${ }^{1}$ Corresponding Author. E-mail: yitingduan@163.com
} 


\section{Methods}

This paper mainly adopts psycho-physical research methods to study the influence of textures on human emotions.

In the first stage, four common medical instruments were selected: dental chair, medical X-ray machine, CT machine and MRI machine, as shown in Figure 1. Five groups of words were used: vivid-boring, warm-cold, soft-hard, relaxed-tense, comfortable-depressing. People's use of these medical devices and their psychological feelings were collected through questionnaires. The age limit for respondents to this experiment was 18-30 years and 43 results were collected. By analyzing the literature on color emotion $^{[7]-[9]}, 32$ groups of words that appear most frequently in the literature were selected, divided and categorized into two groups, one for adjectival words that address the appearance of medical equipment and the other for psycho-emotional words that people use with medical equipment, which are listed separately in Table 1 . In the questionnaire, two questions are set for each of the four groups of medical devices, and respondents can choose the appropriate adjective based on the stem of the question.

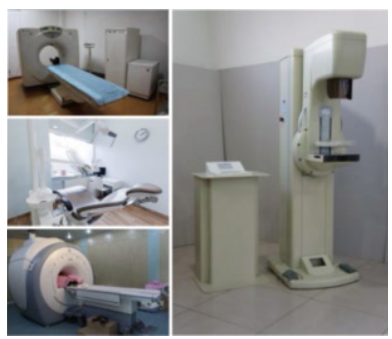

Figure 1. Four common types of medical equipment

Table 1. The two sets of emotive words screened by the experiment

\begin{tabular}{|l|l|}
\hline $\begin{array}{l}\text { Adjectives for the appearance of medical } \\
\text { equipment }\end{array}$ & $\begin{array}{l}\text { Psycho-emotional words for people using } \\
\text { medical equipment }\end{array}$ \\
\hline $\begin{array}{l}\text { light-heavy, hard-soft, cold-hot, bright-dark, } \\
\text { strong-weak, big-small, empty-full, masculine- } \\
\text { feminine, fresh-old, muddy-transparent, modern- } \\
\text { classical, clean-dirty, fashionable-plain, complex- } \\
\text { simple, striking-soft, vivid-dull, elegant-vulgar, } \\
\text { valuable-worthless }\end{array}$ & $\begin{array}{l}\text { beautiful-ugly, humorous-serious, positive- } \\
\text { negative, happy-sad, warm-cold, interesting- } \\
\text { boring, calm-restless, safe-dangerous, stable- } \\
\text { unstable, reliable-unreliable, like-dislike, } \\
\text { harmonious-disharmonious, familiar- } \\
\text { unfamiliar, static-vibrant }\end{array}$ \\
\hline
\end{tabular}

According to the psycho-physical experiments in the first stage, the author screened three groups of words that were chosen most often by the respondents: safety-danger, calm-restlessness, and reliable-unreliable. At the same time, six common textures are selected: glass, fabric, marble, metal, wood and plastic to explore their psychological impact on people. In the second stage, physical objects with corresponding textures were used for the follow-up study of this stage. A total of 54 valid questionnaires were received in this stage, of which 18 were male and 36 were female. It was divided into three experiments: In the first visual experiment, the author placed six kinds of objects in a standard light box to take photos, and adjusted the colors to gray level, as shown in Figure 2. Subjects ranked three pairs of emotional words by observing photos with six kinds of textures. 


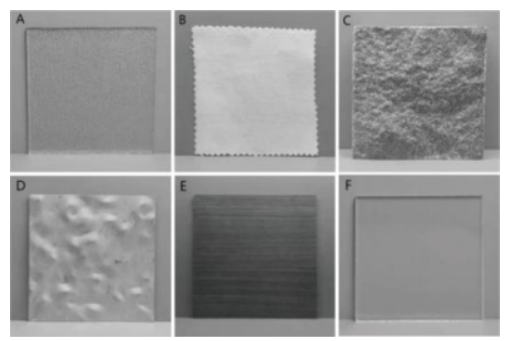

Figure 2. Photographs of six types of textures

The second is the tactile experiment, in which the subjects ranked three pairs of emotional words by touching objects with six textures. The experimental process is shown in Figure 3.

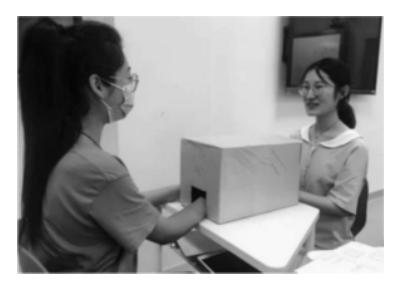

Figure 3. Experimental procedure

The third auditory experiment was conducted, whereby the author recited "A glass, $\mathrm{B}$ fabric, C marble, D metal, E wood and F plastic" in order, and the subjects ranked three pairs of emotional words after listening.

\section{Data Results and Analysis}

\subsection{Safe-dangerous}

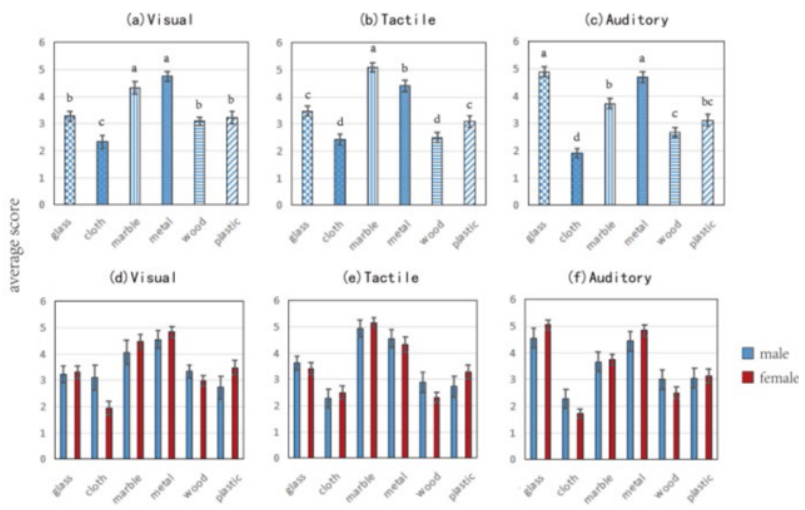

Figure 4. (a)-(c): Data analysis of six types of textures on safe-dangerous in visual, tactile and auditory experiments. (d)-(f): Data analysis of the six types of textures by gender in the visual, tactile and auditory experiments on safe-dangerous. Letters a-d refer to data sets shown to be significantly different to each other (at the $\mathrm{P}=0.05$ level). 
In the visual experiment, as shown in Figure 4 (a), the subjects considered fabric as significantly the safest texture $(\mathrm{P}<0.05)$, marble and metal were significantly the most dangerous textures $(\mathrm{P}<0.05)$, and metal was more dangerous than marble. There was no significant differences among glass, wood and plastic textures $(\mathrm{P}>0.05)$. As shown in figure 4 (d), men considered glass, fabric, wood and plastic as relatively safe textures. Women's cognition of fabric texture safety is significantly higher than that of men.

In the tactile experiment, as shown in Figure 4 (b), subjects considered fabric and wood as significantly the safest textures $(\mathrm{P}<0.05)$, marble was significantly the most dangerous texture $(\mathrm{P}<0.05)$. Glass and plastic were significantly safer textures in subjects' cognition, and there were no significant differences between the two textures $(\mathrm{P}>0.05)$. The data in Figure 4 (e) shows that the cognition of the safety degree of plastic texture and the danger degree of marble texture were significantly higher in males than in females.

In the auditory experiment, as shown in Figure 4 (c), the subjects considered fabric as significantly the safest texture $(\mathrm{P}<0.05)$, glass and metal were significantly the most dangerous textures $(\mathrm{P}<0.05)$. The data of in Figure 4 ( $\mathrm{f})$ shows that males and females have roughly the same cognition and general trend towards the safety-danger degree of six kinds of textures.

Combined with the data in Figure 4, participants ranked the safety-danger degree of the six textures more clearly in the tactile experiment than in the visual and auditory experiments. Except in the tactile experiment the differences between fabric and wood texture were not significant $(\mathrm{P}>0.05)$, the fabric showed significant differences with other textures $(\mathrm{P}<0.05)$. In the visual, tactile and auditory experiments, there were significant differences among all pairs of textures $(\mathrm{P}<0.05)$, indicating that subjects had great cognitive differences in the safe-danger degree of each pair of textures in the three experiments.

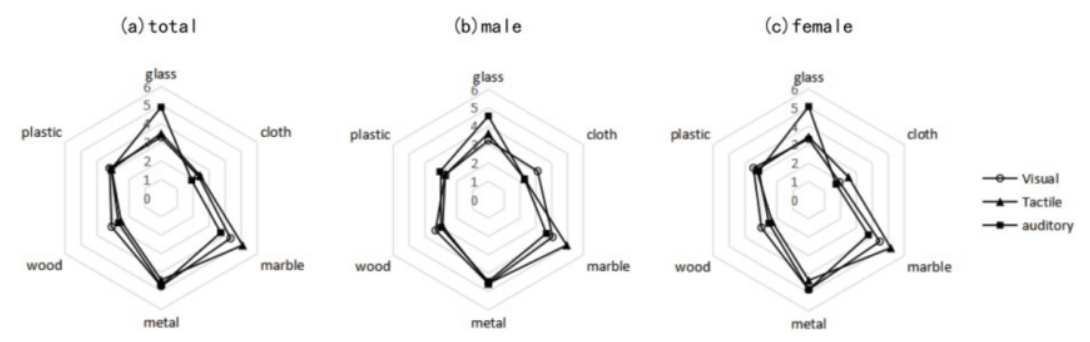

Figure 5. Data analysis of six types of textures regarding safe-dangerous in visual, tactile and auditory experiments.

In the safety-danger emotion ranking, as shown in Figure 5 (a), the subjects thought that glass texture was more significant in danger in auditory experiment than in visual and tactile experiment. Marble textures had different levels of danger in different experiments, where tactile experiment $>$ visual experiment $>$ auditory experiment.

In Figure 5 (b) and (c), male subjects were more likely to perceive the safety of fabric texture in the visual experiment, while female subjects thought that the fabric texture was safer in the tactile experiment.

Based on the data in Figure 4 and Figure 5, in different experiments, fabric was consistently considered to be the safest texture, marble and metal were the more dangerous textures. Compared with the visual and tactile experiments, subjects' 
perceptions of the safety-danger degree of glass and marble textures differed significantly. Glass texture became the most dangerous in the subjects' cognition of texture in the auditory experiment, and women's perception of glass dangerous levels is greater than men, it may be because women will associate glass with sharp objects while hearing it. the participants' perception of the danger degree of marble was significantly lower than that of the visual and tactile experiments, which may be related to the fact that when the subjects heard the word "marble", they would associate it with hardness and other morphological characteristics.

\subsection{Calm-restlessness}

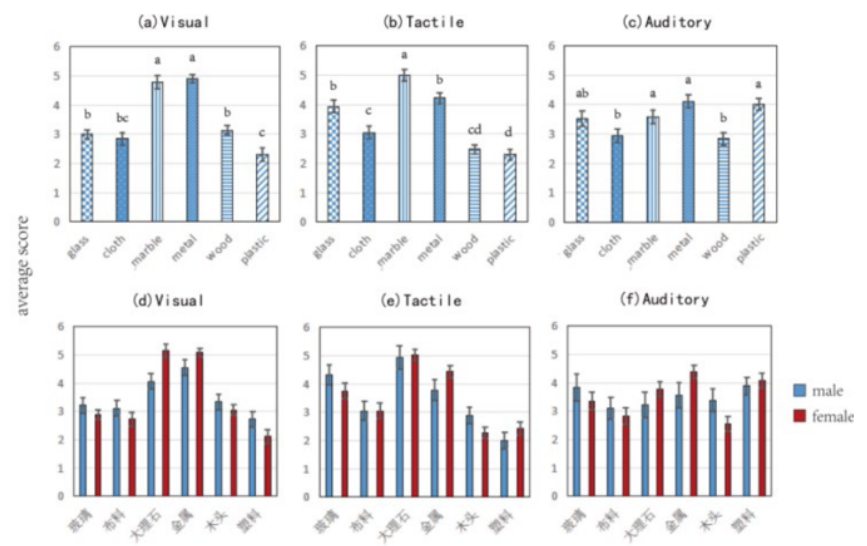

Figure 6. (a)-(c): Data analysis of six types of textures on calm-restlessness in visual, tactile and auditory experiments. (d)-(f): Data analysis of the six types of textures by gender in the visual, tactile and auditory experiments on calm-restlessness. Letters a-d refer to data sets shown to be significantly different to each other (at the $\mathrm{P}=0.05$ level).

In the visual experiment, as shown in Figure 6 (a), the subjects thought that plastic was significantly the calmest texture $(\mathrm{P}<0.05)$, marble and metal were significantly the most restless textures $(\mathrm{P}<0.05)$. There was no significant differences among fabric, glass and wood textures $(\mathrm{P}>0.05)$, also between fabric and plastic texture $(\mathrm{P}>0.05)$. As shown in Figure $6(\mathrm{~d})$, compared with marble texture, men think metal texture is more restless; The cognition of the restlessness degree of marble and metal texture and the calm degree of plastic texture were significantly higher in females than in males.

In the tactile experiment, as shown in Figure $6(\mathrm{~b})$, the subjects thought that plastic was significantly the calmest texture $(\mathrm{P}<0.05)$, metal was significantly the most restless texture $(\mathrm{P}<0.05)$. Wood and fabric were the more calm textures, there were no significant differences between these two textures $(\mathrm{P}>0.05)$, also no significant difference between wood and plastic textures $(\mathrm{P}>0.05)$. The subjects considered glass and metal textures to be more restless, and there were no significant differences between the two textures $(\mathrm{P}>0.05)$. The data in Figure 6 (e) show that women considered the calmness of wood texture similar to that of plastic, and metal texture was more restless than glass texture, while men consider glass texture was more restless than metal texture.

In the auditory experiment, as shown in Figure 6 (c), subjects considered fabric and wood to be significantly the calmest texture $(\mathrm{P}<0.05)$, marble, metal and plastic to be 
significantly the most restless texture $(\mathrm{P}<0.05)$, and the calm and restlessness degree of glass texture was between the two. As shown in Figure 6 (f), males showed no significant differences in the cognitive trend of the calm and restlessness of the six textures, while females believed that wood was significantly the calmest texture and metal was significantly the most restless one. Women's cognition of wood texture calmness and metal texture restlessness were significantly higher than men's.

Combined with the data in Figure 6, participants ranked the calm and restlessness of the six textures more clearly in the tactile experiment than in the visual and auditory experiments. There were no significant differences between fabric and wood texture in visual, tactile and auditory experiments $(\mathrm{P}>0.05)$, indicating that in the cognition of subjects, fabric and wood texture had similar calm and restlessness degree values. In the tactile experiment, the significant effects of each pair of textures were more significant $(\mathrm{P}<0.05)$, indicating that in the tactile experiment, subjects' cognition of the calm and restlessness of each pair of textures was significantly different. Compared with the tactile experiment, in the auditory experiment, the significant effect of each pair of texture was insignificant $(\mathrm{P}>0.05)$, indicating that in the auditory experiment, the subjects had little cognitive differences in the calm and restlessness degree of each pair of textures.

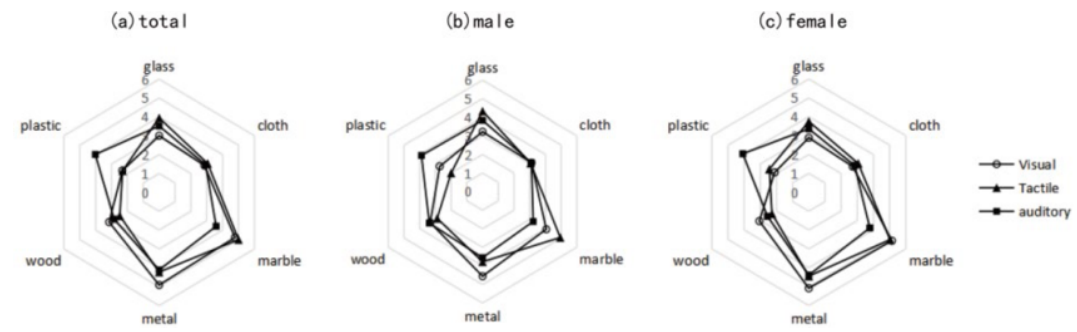

Figure 7. Data analysis of six types of textures regarding safe-dangerous in visual, tactile and auditory experiments.

In the ranking of calm and restlessness, as shown in Figure 7 (a), different from the visual and tactile experiments in which plastic texture was considered to be the calmest texture, the subjects thought that plastic texture showed more significant restlessness in the auditory experiment. The restlessness of marble texture in auditory experiment was significantly lower than that in tactile experiment and visual experiment. The restlessness degree of metal texture and the calm degree of glass texture in visual experiment were significantly higher than those in tactile experiment and auditory experiment.

As shown in Figure 7 (b) and (c), compared with females, males showed more obvious differences in the cognitive trend of calm and restlessness of six textures in visual, tactile and auditory experiments. Men thought that the restlessness of plastic texture was significantly higher in the visual experiment than in the tactile experiment, and the restlessness of marble texture was significantly lower than that in the tactile experiment. Women's cognition of the calm and restlessness of the six textures in different experiments was basically consistent with the general trend.

Based on the data in Figure 6 and Figure 7, in different experiments, metal was consistently considered to be the most restless texture. In the visual and tactile experiments, the plastic texture showed significant calm, but in the auditory experiments, plastic became more restless in subjects perception, this might because subjects in visual and tactile experiments, saw and touched a relatively smooth surface of the acrylic board. In the auditory experiment, the participants' cognition of the degree of marble 
restlessness was significantly lower than that in the visual and tactile experiments, which may be related to the fact that when the subjects heard the word "marble", they would associate it with hardness and other morphological characteristics. In the three experiments, men's cognition of marble calm and restlessness was different, shown as tactile $>$ visual $>$ auditory, which may be related to men's perception of uneven texture on marble surface in the tactile experiment than in the visual experiment, and men thought such uneven texture was more restless. Compared with men, women significantly rated marble and metal as relatively restless in the visual experiment, which may be due to the higher visual perception of these two textures in women than in men.

\subsection{Reliable-unreliable}

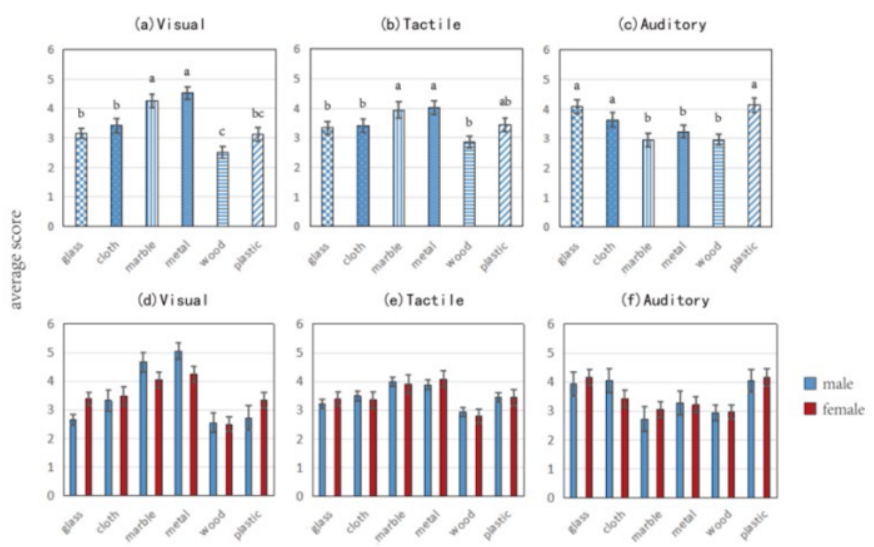

Figure 8. (a)-(c): Data analysis of six types of textures on reliable-unreliable in visual, tactile and auditory experiments. (d)-(f): Data analysis of the six types of textures by gender in the visual, tactile and auditory experiments on reliable-unreliable. Letters a-d refer to data sets shown to be significantly different to each other (at the $\mathrm{P}=0.05$ level).

In the visual experiment, as shown in Figure 8 (a), the subjects considered wood to be significantly the most reliable texture $(\mathrm{P}<0.05)$, marble and metal were significantly the least reliable textures $(\mathrm{P}<0.05)$, and the degree of unreliability of metal was higher than marble. There were no significant differences among plastic, metal and fabric textures $(\mathrm{P}>0.05)$, also no significant differences between wood and plastic textures $(\mathrm{P}>0.05)$. The data in Figure 8 (d) shows that compared with women, men consider glass, wood and plastic textures to be approximately the same level of reliability. Men perceived marble and metal texture to be significantly more unreliable than women.

In the tactile experiment, as shown in Figure 8 (b), subjects considered fabric, metal and wood as significantly the most reliable textures $(\mathrm{P}<0.05)$, and there were no significant differences between the three textures $(\mathrm{P}>0.05)$. Marble and metal were significantly the least reliable textures $(\mathrm{P}<0.05)$, and there was no significant difference between the two textures $(\mathrm{P}>0.05)$. The reliability and unreliability of plastic textures is somewhere in between. The data in Figure 8 (e) shows that male and female subjects have roughly the same cognition of the reliability-unreliability degree of the six textures, which is consistent with the general trend. 
In the auditory experiment, as shown in Figure 8 (c), subjects thought marble, metal and wood were significantly the most reliable textures $(\mathrm{P}<0.05)$, and there were no significant differences among the three textures $(\mathrm{P}>0.05)$; glass, fabric and plastic were significantly the least reliable textures $(\mathrm{P}<0.05)$, and there were no significant differences among the three textures $(\mathrm{P}>0.05)$. The data in figure 8 (f) shows that male and female subjects have roughly the same cognition of the reliability-unreliability degree of the six textures, which is consistent with the general trend.

Combined with the data in Figure 8, in the visual experiment, subjects ranked the reliability and unreliability of the six textures more clearly than those in the tactile and auditory experiments. The differences of glass-fabric, glass-plastic, fabric-plastic and marble-metal textures in visual, tactile and auditory experiments were not significant $(\mathrm{P}>0.05)$, indicating that in the cognitive perception of the subjects, glass-fabric, glassplastic, fabric-plastic and marble-metal textures have similar reliability-unreliability degree values respectively. There were significant differences between glass-metal and fabric-marble textures in visual, tactile and auditory experiments $(\mathrm{P}<0.05)$, which indicated that there were significant differences in subjects' cognition of glass-metal and fabric-marble textures in reliability-unreliability degree. Compared with visual and auditory experiments, in the tactile experiment, the significant effects of each pair of textures were insignificant $(\mathrm{P}>0.05)$, indicating that in the tactile experiment, the cognitive difference between reliability and unreliability of each pair of textures was less, followed by auditory experiment, and finally by visual experiment.

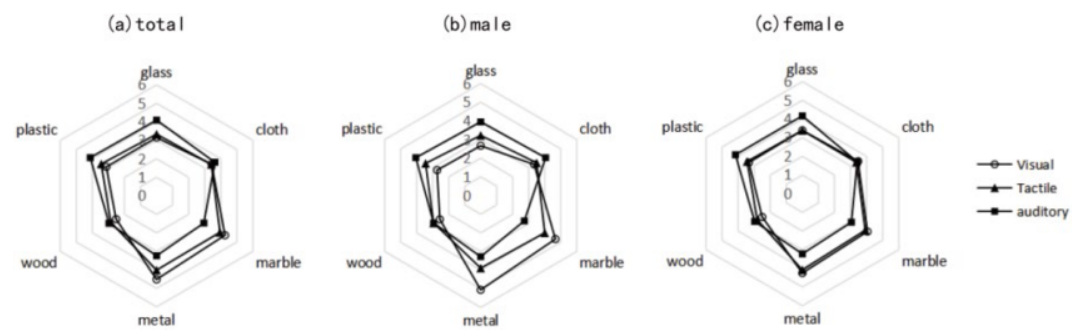

Figure 9. Data analysis of six types of textures regarding reliable-unreliable in visual, tactile and auditory experiments.

In the ranking of reliable-unreliable emotional, as shown in Figure 9 (a), different from the visual and tactile experiment of marble and metal texture considered to be the most unreliable, subjects thought in the auditory experiments, glass and plastic grain showed more significant unreliability, and marble and metal are thought to be relatively reliable textures in the auditory experiments.

In Figure 9 (b) and (c), compared with females, males showed more obvious differences in the cognitive trends of reliability-unreliability of the six textures in visual, tactile and auditory experiments. Men thought marble and metal texture were less reliable and glass and plastic were safer in visual experiments than in tactile experiments. The female's perception of the reliability and unreliability of the six textures in different experiments was basically consistent with the general trend.

Based on the data in Figure 8 and 9, in different experiments, wood was consistently considered to be the most reliable texture. Auditory experiments significantly improved the perception of the unreliability of glass and plastic textures and the perception of the reliability of marble and metal textures, which may be related to the fact that when the subjects heard relevant textures, there is a certain degree of difference between the 
texture features they think of and the texture features they actually see or feel. In reliableunreliable emotional ranking experiment, males' cognition of six kinds of textures in the three experiments significantly differ from that of female, males' different emotional cognition on the same texture in different experiments indicates that the men have different understandings of the same thing in the visual, tactile and auditory sense, that may be related to the fact that male has an active brain thinking ability.

Combined with the data in Figure 5, 7, 9, in the sorting experiment of three pairs of emotional words, the subjects believed that the fabric and wood texture had basically the same cognition in visual, tactile, auditory sense respectively, showing a relatively safe, calm and reliable emotional trend. There were some differences in the emotional perception of different textures between men and women in different experiments. Auditory experiment significantly affected the cognitive degree of the three pairs of emotion words, mainly reflected in the glass, marble, metal and plastic textures, especially in the cognitive degree of safety-danger, reliability-unreliability of the glass texture, and the cognitive degree of calm-restlessness of plastic texture.

\section{Conclusion}

Based on the experimental data, as shown in Figure 10 and Figure 11, wood and fabric are the relatively safe textures in subjects' cognition, metal is the relatively dangerous texture in subjects' cognition, wood is the most reliable texture in subjects' cognition, and fabric and plastic are the relatively unreliable texture in subjects' cognition.

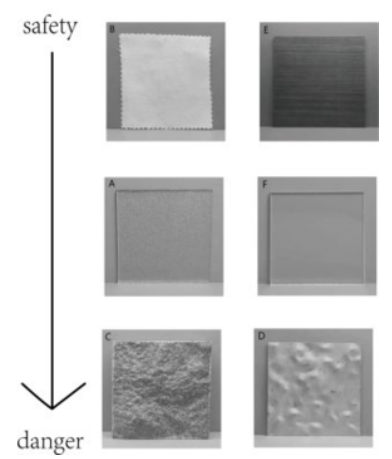

Figure 10. Texture sorting on safety-danger

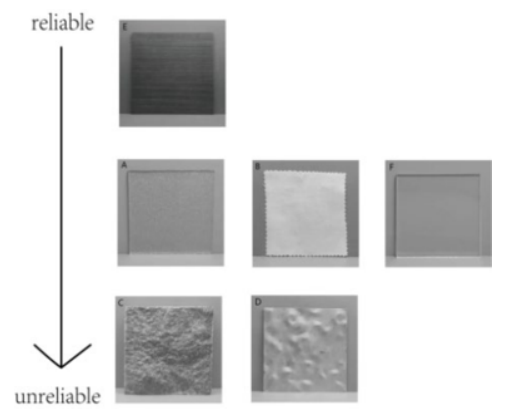

Figure 11. Texture sorting on reliable-unreliable 
With the development of science and technology and economy, people's living standards have been improved more and more, people begin to pay more attention to their own psychological feelings. The author hopes to give people a positive emotional attitude by designing and improving their textures and using appropriate collocation on some special occasions or when using some equipment. For example, producers can change their appearance textures when producing large medical equipment used in hospitals, which may, to some extent, subtly affect the negative emotions of some severe patients like the elderly or children, and increase their confidence and sense of security.

\section{Acknowledgement}

This work was supported by Grant Project: Youth Project of Zhejiang Provincial Natural Science Foundation (No. LQ19C090009)

\section{References}

[1] Reddy SM, Chakrabarti D, Karmakar S. Emotion and interior space design: An ergonomic perspective. Work. 2012; 41(6): 1072.

[2] Akamatsu M, Mackenzie IS, Hasbroucq T. A comparison of tactile, auditory, and visual feedback in a pointing task using a mouse-type device. Ergonomics. 1995; 38(4): 816.

[3] Klatzky RL, Lederman SJ, Metzger VA. Identifying objects by touch: An "expert system". Perception \& Psychophysics. 1985; 37(4): 299.

[4] Jalilzadehazhari E, Johansson J. Material properties of wooden surfaces used in interiors and sensory stimulation. Wood Material Science \& Engineering. 2019; 14(4): 192-193.

[5] Nyrud AQ, Bringslimark T. Is interior wood use psychologically beneficial? A review of psychological responses toward wood. Wood and Fiber Science. 2010; 42(2): 202-203.

[6] Bhatta SR, Kaisa T, Katja V, Mark H, Marketta K. Sensory and Emotional Perception of Wooden Surfaces through Fingertip Touch. Frontiers in Psychology. 2017; 8: 367.

[7] Kobbayashi S. The Aim and Method of the Color Image Scale. Color Research and Application. 1981; 6: 93-107.

[8] Hogg J. A Principal Component Analysis of Semantic Differential Judgements of Single Colors and Color Pairs. Journal of General Psychology. 1969; 88: 129-140.

[9] Ou LC, Luo M R, Sun P L, Hu N C and Chen H S. Age Effects on color Emotion, Preference, and Harmony. Color Research and Application, 2012, 37: 92-105. 